\title{
Erratum to: Editorial
}

Chang-Jiu Li, Seiji Kuroda, Masahiro Fukumoto, Khiam Aik Khor, Changhee Lee, and You Wang

\section{Erratum to: $\mathrm{J}$ Therm Spray Tech DOI 10.1007/s11666-010-9559-z}

The family name of Guest Editor Changhee Lee was misspelled as "Li" in the original article. Please note that the correct spelling of his family name is "Lee" as shown in this erratum.

The online version of the original article can be found under doi:10.1007/s11666-010-9559-z.

Chang-Jiu Li, Xi'an Jiaotong University, Xi’an, Shaanxi, China; Seiji Kuroda, National Institute of Materials Science, Tsukuba, Ibaraki, Japan; Masahiro Fukumoto, Toyohashi University of Technology, Toyohashi, Aichi, Japan; Khiam Aik Khor, Nanyang Technological University, Singapore, Singapore; Changhee Lee, Hanyang University, Seoul, Korea; and You Wang, Harbin Institute of Technology, Harbin, Heilongjiang, China. 